\title{
Optimal methodology for distribution systems reconfiguration based on OPF and solved by decomposition technique
}

\author{
H. M. Khodr, J. Martínez-Crespo, Z. A. Vale and C. Ramos
}

\begin{abstract}
SUMMARY
This paper presents a new and efficient methodology for distribution network reconfiguration integrated with optimal power flow (OPF) based on a Benders decomposition approach. The objective minimizes power losses, balancing load among feeders and subject to constraints: capacity limit of branches, minimum and maximum power limits of substations or distributed generators, minimum deviation of bus voltages and radial optimal operation of networks. The Generalized Benders decomposition algorithm is applied to solve the problem. The formulation can be embedded under two stages; the first one is the Master problem and is formulated as a mixed integer non-linear programming problem. This stage determines the radial topology of the distribution network. The second stage is the Slave problem and is formulated as a non-linear programming problem. This stage is used to determine the feasibility of the Master problem solution by means of an OPF and provides information to formulate the linear Benders cuts that connect both problems. The model is programmed in GAMS. The effectiveness of the proposal is demonstrated through two examples extracted from the literature.
\end{abstract}

Supporting information may be found in the online version of this article.

key words: Benders decomposition; distribution system; optimal power flow; optimization; reconfiguration

\section{INTRODUCTION}

Distribution networks are normally meshed in design but the operation is nearly always configured radially. Their configurations may be varied with manual or automatic switching operations for the loads supplied at the minimum cost of possible line losses, increasing system security and enhancing power quality [1]. Reconfiguration consists of changing the network configuration by opening/closing feeders and tie-switches so that the networks become radial in operation.

A great deal of research has been conducted regarding loss minimization in the area of network reconfiguration of distribution systems [2-7], but few studies have jointly tried the theme of reconfiguration with optimal power flow, using a deterministic optimization technique in a single joint model.

Distribution system reconfiguration (DSR) for loss reduction was studied by Merlin and Back [3]. Their solution scheme starts with a meshed distribution system obtained by considering all switches closed and then the switches are opened successively to eliminate the loops. An equivalent linear resistive network model is used to determine the branch to be opened. Shirmohammadi and Hong [4] have suggested a heuristic algorithm based on the Merlin and Back method [3]. Here also, the solution procedure starts by closing all the network switches, which are opened one after another so as to establish the optimum flow pattern in the networks using many approximations. Borozan et al. [5] have presented a method similar to Reference [4], however, this method contains three main parts: load estimation, effective determination of power losses configuration and cost/benefit evaluation. Civanlar $e t$ al. [6] made use of heuristics and the Branch Exchange technique to determine a distribution system configuration which would reduce line losses. Baran and $\mathrm{Wu}$ [7] have made an attempt to improve the method of Civanlar et al. [6] by introducing two approximation 
formulas for calculating power flow in the transfer of system loads. Kashem et al. [8] have proposed a branch exchange method for network reconfiguration. This is basically an extensive search method which considers all the tie-switches. Chen and Cho [9] have performed an analysis of an hourly reconfiguration schedule. They have studied the hourly load patterns over an interval of a year in order to define the hourly load conditions for each season. They have used the branch and bound technique for obtaining a minimum losses configuration. Nara et al. [10] have proposed a method of distribution system reconfiguration for reduction of real power losses using a genetic algorithm. Lin et al. [11] have applied a refined genetic algorithm to the network reconfiguration problem in order to reduce resistive line losses. Huang [12] has proposed a genetic algorithm based on a fuzzy approach for network reconfiguration of distribution systems. Although the researchers [10-12] have demonstrated the effectiveness of genetic algorithms for network reconfiguration, solution time is highly prohibitive. Lin and Chin [13,14] have presented an algorithm for distribution feeder reconfiguration. They have used a voltage index, an ohmic index and a decision index to determine the switching operation. Huang and Chin [15] have proposed an algorithm based on a fuzzy operation to deal with the feeders reconfiguration problem. Their approach tries to minimize power losses and acquire the load balance at the same time. Liu et al. [16], Jung et al. [17] and Auguliaro et al. [18] have proposed applications based on artificial intelligence for a minimum losses configuration. Hsiao [19] has proposed a fuzzy multi-objective technique based on an evolution programming method for network reconfiguration.

Matos et al. [20-22] present an approach to the reconfiguration of radial distribution networks for both loss reduction and service restoration, using the simulated annealing meta-heuristic technique. They minimize the non-supplied load, and the total number of switching operations is included as a possible criterion. In Reference [21], the same authors consider a set of load scenarios and find single or multiple configuration solutions that minimize both energy losses and switching actions in a bi-criteria framework, using the same meta-heuristic technique.

Pereira et al. [23] present a model for identifying optimal operation strategies of electric distribution networks, minimizing active power losses and taking into account the transformers taps and the capacitor banks that are in operation. This problem is solved by a simulated annealing meta-heuristic technique and tested on a realistic problem based on a Portuguese distribution system.

The DSR and OPF problems have been addressed and studied separately by many works reported in the literature, but they have scarcely been studied at the same time applied to the same model and using in the calculation process deterministic optimization techniques or the Benders decomposition optimization technique. This innovative approach of the Benders decomposition algorithm had not been previously used in distribution system reconfiguration problems. References [1-23] are dedicated to the reconfiguration problem, whereas Reference [24] applies the OPF to distribution system reconfiguration independently.

Gomes et al. [25] present a heuristic approach for DSR based on OPF in which the branches' status (open/closed) is presented by continuous functions. In the approach, all branches are initially considered closed, and from the OPF results, a heuristic technique is used to determine the next loop to be broken by opening one switch. Then, the list of switches that are candidates to be opened is updated, and the aforementioned process is repeated until all loops are broken, making the distribution system radial. This method cannot guarantee a global optimal solution of the DSR problem as it will be seen next.

The formulations differ in each one due to the representation in the model of fewer or more problem features and/or the use of different solution algorithms. The problem is such that the more features that are modeled, the higher the required computational effort is. Most of the research studies consider the formulation problem as two separate problems; the first one consists of the optimal DSR through the divers' method and the second is the OPF. This paper considers the two problems to be the same one and it is solved jointly by the Benders decomposition algorithm [26,27], which has already been applied to solve different problems in electric power systems [28-31].

Optimal power flow has been extensively used in power systems at the generation/transmission level to designate the problem of finding the optimal value for the control variable (real and reactive power, voltage settings, capacitors banks set points, and taps.) when minimizing the total operation cost, while respecting the technical constraints of the network and equipments. In distribution systems, many approaches exist dedicated to the optimization of the configuration and the optimization of the voltage profile through capacitors banks. In both cases the objective function seeks for losses reduction. Moreover, the recent development of distribution systems has led to the presence of distributed generation that introduces uncertainty in the previously mentioned problems, but may contribute to voltage control and optimization. In the same cases, regional dispatch of this kind of units is feasible, turning it possible to seek the optimal operating policies.

Although it still is little introduced in the surveyed literature, the recent most important feature of loss minimization or load balancing problem is the existence of distributed generators (DG) in a distribution system. The loss minimum or load balancing reconfiguration may become difficult since DGs are normally operated by customers, and power supplier cannot have any generation information of DGs. Moreover, three-phase load balancing may be an emerging problem if lots of single phase DGs are installed in demand side. 
This paper introduces the concept of distribution optimal power flow (DOPF), while aims at integrating all the decision problems in an unified model where reconfiguration, distributed generation and capacitors banks are used to find the optimal operating point for the distribution network.

The DSR and DOPF problem for the distribution system loss minimum reconfiguration has been formulated by means of Benders decomposition and divided in two stages, named Master and Slave levels. The decision problem or Master problem is formulated as a mixed integer non-linear programming problem (MINLP) and determines the radial topology of the distribution network. The second stage is the Slave problem and is formulated as a non-linear programming problem (NLP). This stage is used to define the feasibility of the Master problem solution by means of an OPF and provides information with which to formulate the linear Benders cuts that transfer information from the Slave to the Master problem.

It is shown in this paper that the feasibility seeking variant of the Benders algorithm using implicit enumeration is very efficient for solving reconfiguration and optimal power flow of large-scale distribution systems with very short computational time, and improves the results provided previously in scientific literature.

In this paper the proposed methodology is programmed in General Algebraic Modeling System (GAMS) that is a high-level modeling language for mathematical programming and optimization. It consists of a language compiler and a stable of integrated high-performance solvers (e.g., CPLEX for linear, mixed-integer linear or mixed-integer quadratic programming, CONOPT for non-linear programming... .). Other commercial languages with similar features are AMPL, AIMMS, or XPRESS-MO, but GAMS is probably the most widespread one. The optimization problems are modeled with GAMS in a highly compact and natural way. GAMS allows you to build models that can be adapted quickly to new changes, can even convert from linear to mixed-integer or nonlinear with little trouble, and can change from one solver to another one. The main disadvantage of GAMS is that it needs time for model creation and interface with the solver that slows down the obtaining of results, but it can only be critical with very largescale optimization models, which it is not our case.

As was previously mentioned, the model is programmed in GAMS [32], using the solver CPLEX [33] for solving the MINLP problem with a quadratic objective function (Master level) and CONOPT [34] for solving the NLP problem (Slave level). The algorithm will solve the distribution reconfiguration integrated with an OPF for a single-phase or balanced three-phase AC network. The algorithm could be also modified to handle unbalanced three-phase by treating each current quantity as a vector containing either the line currents or the magnitudes of their symmetrical components. A symmetrical-component representation would be necessary if the line impedance depended significantly on phase sequence, but this dependence is generally much less for resistance than for reactance quantities.

If the distribution system data per phase is available, this methodology can be executed per phase for solving unbalanced distribution systems reconfiguration.

It should be noticed that the results do not depend on modeling language (GAMS). These results would be the same using other programming language platform.

This paper is structured as follows. Section 2 deals with the optimal networks reconfiguration problem, defines the model equations, and briefly describes the method applied to solve the problem, an approach to the Generalized Benders decomposition, defining the Master and Slave problems. In both problems, the set of constraints and the objective function are listed. In Section 3 , the solution procedure for the Slave and Master problems is detailed. Furthermore, this section illustrates most of the advantages and potentialities of the proposed model using two study cases extracted from References [1] and [35]. Finally, Section 4 states the conclusions.

\section{PROBLEM FORMULATION}

The reconfiguration integrated with optimal power flow (ROPF) model must be able to determine the optimal configuration and evaluate the load distribution amongst substations, distributed generators and feeders. The resulting system should be feasible, i.e., it should meet demand and technical requirements. There is a considerable amount of feasible alternatives and the model chooses the alternative that optimizes a specific objective function.

In this paper, the objective function of the ROPF model seeks to minimize the system power losses. The set of constraints comprises: power balance constraints-Kirchhoff's 1st Law; capacity limits of the existing substations, distributed generators and feeders; voltage calculation constraints-Kirchhoff's 2nd law; voltage limits for all load buses; radiality constraints; logical constraints - involving integer decision variables - and all the OPF constraints. The ROPF formulation is stated as follows. 
The objective function seeks to minimize power losses:

$$
\operatorname{Min} Z=\sum_{i \in N} \sum_{k \in \Phi_{i}} C_{k}^{i} S_{k}^{i^{2}}
$$

Subject to the following constraints:

Kirchhoff's first law (power balance) in the buses:

$$
\sum_{k \in \Phi_{i}^{\mathrm{IN}}} S_{k}^{i}-\sum_{l \in \Phi_{i}^{\mathrm{out}}} S_{l}^{i}=S_{i} ; \quad \forall i \in N_{d}
$$

Minimum power output limits of the substation or generator:

$$
\sum_{k \in \Phi_{p}^{\mathrm{OUT}}} S_{k}^{p} \geq \sum_{t \in \Psi_{p}^{\prime}} S_{t, p}^{\min } \cdot y_{t}^{p} ; \quad \forall p \in N_{g}
$$

Maximum power output limits of the substation or generator:

$$
\sum_{k \in \Phi_{p}^{\text {oUT }}} S_{k}^{p} \leq \sum_{t \in \Psi_{p}^{\prime}} S_{t, p}^{\max } \cdot y_{t}^{p} ; \quad \forall p \in N_{g}
$$

Power limits of the line or thermal limits of the line:

$$
S_{l} \leq S_{l, i}^{\max } \cdot w_{l}^{i} ; \quad \forall l \in \Phi_{i}, \forall i \in N
$$

Radial operation condition of the networks:

$$
\sum_{l \in \Phi_{i}^{I N}} w_{l}^{i} \leq 1 ; \quad \forall i \in N
$$

Between two buses, the line is represented by double direction of the power flow. In the operation of the networks, only one of the directions should exist.

General power balance in the networks:

$$
\sum_{i \in N} \sum_{k \in \Phi_{i}^{\text {OUT }}} S_{k}^{i} \leq \sum_{p \in N} \sum_{t \in \Psi_{p}^{\prime}} S_{t, p}^{\max } \cdot y_{t}^{p}
$$

Real power output limits:

$$
y_{t}^{p} \cdot P_{t}^{\min } \leq P_{t}^{p} \leq P_{t}^{\max } \cdot y_{t}^{p} ; \quad \forall t \in \Psi_{p}, \forall p \in N_{g}
$$

Reactive power output limits:

$$
y_{t}^{p} \cdot Q_{t}^{\min } \leq Q_{t}^{p} \leq Q_{t}^{\max } \cdot y_{t}^{p} ; \quad \forall t \in \Psi_{p}, \forall p \in N_{g}
$$

Reactive power output by shunt capacitors:

$$
\begin{array}{ll}
Q_{r}^{n}=B_{r, b}^{n} \cdot y_{r, b}^{n} \cdot\left(V_{n}\right)^{2} & \forall r \in \Omega_{c}, n \in N_{d r}, b \in B \\
\sum_{b \in B} y_{r, b}^{n} \leq 1 ; & \forall r \in \Omega_{c}, n \in N_{d r}
\end{array}
$$

Real power flow equations:

$$
\sum_{n \in N} V_{i} \cdot V_{n} \cdot\left(G_{\text {in }} \cdot \cos \left(\delta_{i}-\delta_{n}\right)+B_{\text {in }} \cdot \sin \left(\delta_{i}-\delta_{n}\right)\right)=P_{i}^{g}-P_{i}^{d} ; \quad \forall i \in N_{d}: P_{i}^{g}=\sum_{t \in \Psi_{i}} P_{t}^{i}
$$


Reactive power flow equations:

$$
\sum_{n \in N} V_{i} \cdot V_{n} \cdot\left(G_{\mathrm{in}} \cdot \sin \left(\delta_{i}-\delta_{n}\right)-B_{\mathrm{in}} \cdot \cos \left(\delta_{i}-\delta_{n}\right)\right)=Q_{i}^{g}-Q_{i}^{d} ; \quad \forall i \in N_{d}: Q_{i}^{g}=\sum_{i \in \Psi_{i}} Q_{t}^{i}
$$

Capacity limits of distribution lines:

$$
\left|V_{i} \cdot\left[\left(V_{i}-V_{n}\right) \cdot y_{\mathrm{in}}\right]^{*}+V_{i} \cdot\left(V_{i} \cdot\left(\frac{1}{2} \cdot y_{\mathrm{in}}^{\prime}\right){ }^{*}\right)\right| \leq S_{\mathrm{in}}^{\max } ; \quad \forall i, n \in N:(\text { in }=l) \in \Phi_{i}
$$

Bus voltage magnitude limits:

$$
V^{\min } \leq V_{n} \leq V^{\max } ; \quad \forall n \in N
$$

Bus angle limits:

$$
-\pi \leq \delta_{n} \leq \pi \quad \forall n \in N /\left\{n_{s}\right\} ; \quad \delta_{n s}=0 ; \quad n_{S} \text { : swing bus }
$$

Transformer taps limits:

$$
\mathrm{RT}_{\mathrm{in}}^{\min } \leq r t_{\mathrm{in}} \leq \mathrm{RT}_{\mathrm{in}}^{\max } ; \quad \forall n, i \in N:(\text { in }) \in \Psi_{i} .
$$

\subsection{Benders decomposition}

The ROPF problem addressed in this paper is formulated as a MINLP problem with a non-linear objective function, binary decision variables, continuous variables for operation processes, and non-linear constraints such as complete power flow equations or distribution lines capacity limits.

The difficulties related to solving non-linear optimization problems with binary variables force us to make use of partitioning techniques such as the Benders decomposition [26,27]. The Benders partition algorithm is a decomposition technique on two-levels, Master and Slave, which defines an iterative procedure between both levels in order to reach the joint optimal solution. The master level represents the decision problem, which is defined as a MINLP problem, whereas the Slave level deals with the operation problem, being a non-linear OPF. This method allows us to appropriately treat the non-convexity associated with binary variables and to divide the global problem into two smaller problems which are easier to solve.

The Master problem determines the new configuration of the network and the open switches, as well the on/off status of capacitors and generators by means of Branch and Bound optimization technique using the solver CPLEX [33]. This solution obtained by CPLEX is transferred to the Slave sub-problem, which verifies the technical feasibility of the Master problem solution by means of OPF that can be solved using CONOPT [34]. It defines the dual values (sensitivities) associated with the configuration decision made previously by the Master problem. In the iterative process, this information is supplied to the Master problem through the Benders cuts in order to improve the new decision of the Master problem in the next iteration.

The procedure followed in this paper includes the steps illustrated in the flowchart in Figure 1. The "start" point will be the initial configuration of the network.

\subsection{Master problem}

The Master problem decides the network configuration in order to minimize the system power losses by opening/closing tieswitches (1/0 binary variables) so that the network becomes radial in operation, and also by connecting/disconnecting capacitors and/or generating units. Therefore, all binary variables have to be included in the optimization problem of this level.

The objective function minimizes:

$$
\sum_{i \in N} \sum_{k \in \Phi_{i}} C_{k}^{i} S_{k}^{i^{2}}+\alpha^{*}
$$




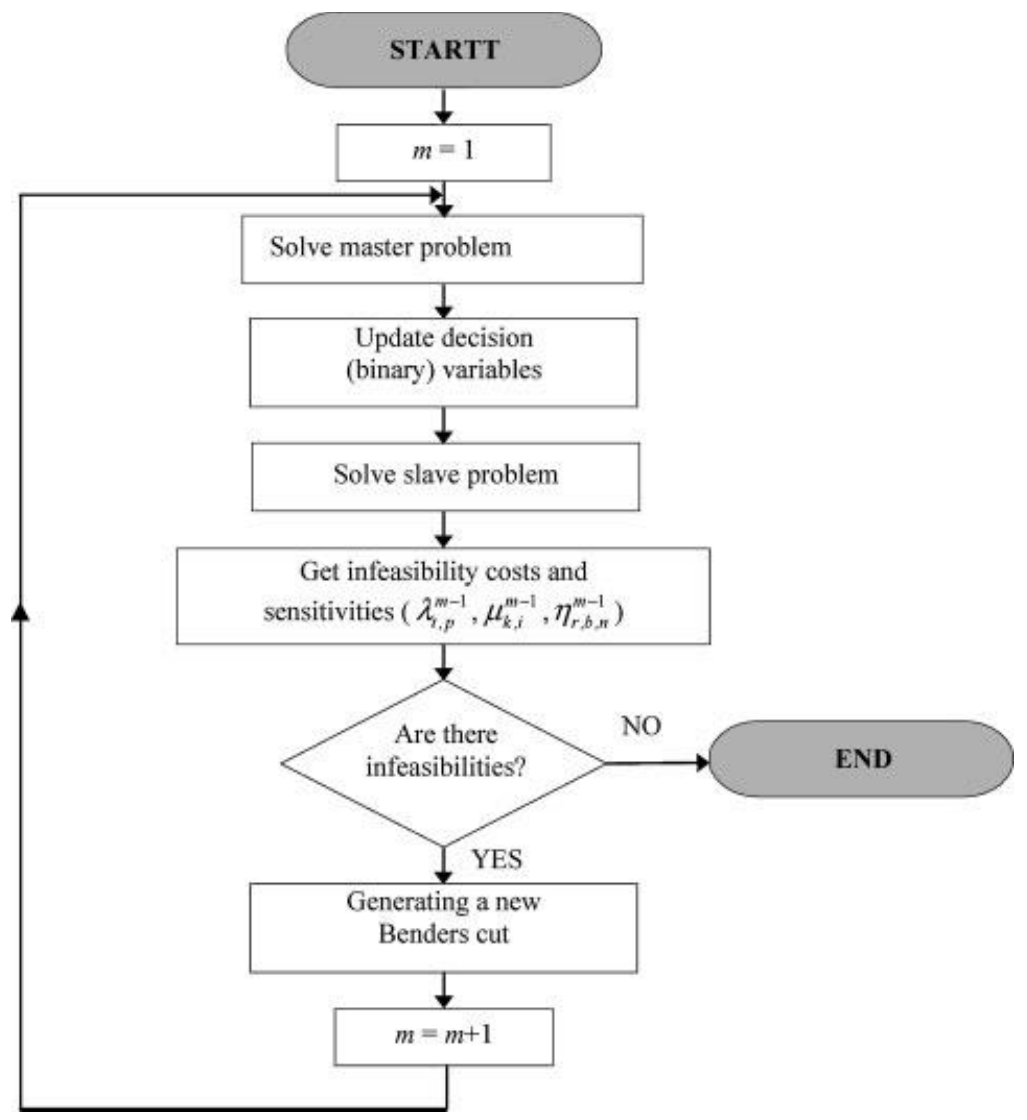

Figure 1. Benders decomposition flowchart.

Subject to the constraints (2)-(9) and the Benders cuts:

$$
\begin{aligned}
\alpha^{*} & \geq \alpha\left(Y_{t, p}^{m-1}, W_{i, k}^{m-1}, Y_{r, b, n}^{m-1}\right)+\sum_{p \in N_{g}} \sum_{t \in \Psi_{p}} \lambda_{t, p}^{m-1} \cdot\left(y_{t}^{p}-Y_{t, p}^{m-1}\right)+\sum_{k \in \Psi_{i}} \sum_{i \in N} \mu_{k, i}^{m-1} \cdot\left(w_{k}^{i}-W_{k, p}^{i, m-1}\right) \\
& +\sum_{r \in \Omega} \sum_{b \in B} \sum_{n \in N_{d r}} \eta_{r, b, n}^{m-1} \cdot\left(y_{r, b}^{n}-Y_{r, b, n}^{m-1}\right) ; \quad \forall m \in M
\end{aligned}
$$

The first term of the objective function represents the operation cost (power losses cost). The second term means, through the real variable $\alpha^{*}$, an underestimation of the Slave sub-problem costs or unfeasibility costs. Therefore, the optimization variables of this problem are $\alpha^{*}, S_{k}^{i}$, and the connection binary variables of transformers or generator $\left(y_{t}^{p}\right)$, capacitors $\left(y_{r, b}^{n}\right)$ and branches/feeders $\left(w_{k}^{i}\right)$.

The key issue in Benders decomposition is located at Equations (18), the Benders linear cuts, which are feasibility cuts in this problem. The levels, Master and Slave, are coupled by these cuts which are updated at each iteration. These cuts provide the Master problem with information about the unfeasibility cost of the previous iteration $\left(\alpha^{*}\right)$ and the sensitivities $\left(\lambda_{t, p}^{m-1}, \mu_{k, i}^{m-1}, \eta_{r, b, n}^{m-1}\right)$ associated with the decision taken by the Master problem in the previous iteration $\left(Y_{t, p}^{m-1}, W_{i, k}^{m-1}, Y_{r, b, n}^{m-1}\right)$ (see Equation (20)), which is already known. This information helps the Master problem to make a new decision and reach the optimal solution.

\subsection{Slave sub-problem}

The Slave level checks the feasibility of the Master problem solution and provides the optimal value of the operation variables by means of an AC-OPF. Therefore, the Slave sub-problem solves system operation, minimizing the cost of infeasibility, as there could 
be some cases where the master level solution does not guarantee its feasibility. A way to make the OPF problem always feasible is to add slack values at power flow equations (possible bus voltage violation) or at some lines (possible lines overload) and to include them in the objective function so that the objective function minimizes the infeasibilities of the power system. At the last iteration, the end solution of the global problem has to be feasible and optimal, that is, all these slack variables should be equal to zero.

Therefore, the objective function would be formulated as

$$
\sum_{n \in N}\left(\overline{P_{n}}+\overline{Q_{n}}\right)+\sum_{k \in \Phi_{i}} \overline{S_{k}^{i}}
$$

where $\overline{P_{n}}, \overline{Q_{n}}$, and $\overline{S_{k}^{i}}$ are the slack variables of the optimization problem. They represent, respectively, the requirements of real power, reactive power, and the increment of line power limit necessary to make the optimization problem feasible.

It is subject to constraints (8)-(16) and

$$
\begin{array}{ll}
y_{t}^{p}=Y_{t, p}^{m}: \lambda_{t, p}^{m-1} & \forall t \in \Psi_{p}, \forall p \in N_{g} \\
w_{k}^{i}=W_{k, i}^{m}: \mu_{k, i}^{m-1} & \forall k \in \Phi_{i}, \forall i \in N \\
y_{r, b}^{n}=Y_{r, b, n}^{m}: \eta_{r, b, n}^{m-1} & \forall r \in \Omega_{c}, \forall b \in B, n \in \in n_{d r}
\end{array}
$$

Constraint (20) provides the dual value associated with the decision taken by the Master problem, that is, the sensitivity for each value of the decision variables $y_{t}^{p} ; w_{k}^{i} ; y_{r ; b}^{n}$ fixed by the Master problem at the same iteration. These sensitivities are going to be applied to the formulation of the Benders cuts of the following iteration (see Equation (18)). The cuts couple Master and Slave problems and are updated in each iteration to improve the new decision of the Master problem.

The minimization is subject to the $\mathrm{AC}$ full power flow equations, real and reactive output production limits, reactive power injected by capacitors (see Equation (10)), transmission capacity limits of lines, the bus voltage angle and magnitude limits. Therefore, the Slave problem determines the value of the operation variables $P_{t} ; Q_{t}^{p} ; Q_{r}^{n} ; V_{n} ; \mathrm{d}_{n} ; r t_{\mathrm{in}}$. The control variables of the OPF problem in electrical power systems normally are the real power and the bus voltage magnitude in $P V$ buses, the transformer taps and capacitors/reactors susceptance. In our case, a distribution system, the control variables are the transformer taps, the reactive power by switching capacitor banks and therefore the bus voltage. The active power is controlled by means of the switches (on/off) connected in the distribution networks, so the load can be transferred from a feeder to another.

The slack variables would be included in the Equations (11), (12), and (13) to make feasible the OPF. These constraints would be formulated now as

$$
\begin{aligned}
& \sum_{n \in N} V_{i} \cdot V_{n} \cdot\left(G_{\mathrm{in}} \cdot \cos \left(\delta_{i}-\delta_{n}\right)+B_{\mathrm{in}} \cdot \sin \left(\delta_{i}-\delta_{n}\right)\right)=P_{i}^{g}+\overline{P_{n}}-P_{i}^{d} ; \quad \forall i \in N_{d}: P_{i}^{g}=\sum_{t \in \Psi_{i}} P_{t}^{i} \\
& \sum_{n \in N} V_{i} \cdot V_{n} \cdot\left(G_{\mathrm{in}} \cdot \sin \left(\delta_{i}-\delta_{n}\right)-B_{\mathrm{in}} \cdot \cos \left(\delta_{i}-\delta_{n}\right)\right)=Q_{i}^{g}+\overline{Q_{n}}-Q_{i}^{d} ; \quad \forall i \in N_{d}: Q_{i}^{g}=\sum_{t \in \Psi_{i}} Q_{t}^{i} \\
& \left|V_{i} \cdot\left[\left(V_{i}-V_{n}\right) \cdot y_{\mathrm{in}}\right]^{*}+V_{i} \cdot\left(V_{i} \cdot\left(\frac{1}{2} \cdot y_{\mathrm{in}}^{\prime}\right)^{*}\right)\right|-\overline{S_{\mathrm{in}}^{i}} \leq S_{\mathrm{in}}^{\max } ; \quad \forall i, n \in N:(\text { in }=k) \in \Phi_{i} .
\end{aligned}
$$

\subsection{Benders convergence criterion}

The Benders decomposition procedure stops when the solution provided by the Master problem is feasible, that is, the value of the objective function computed in the Slave problem is zero.

\section{TEST CASES}

To compare the proposed methodology with others published in the literature, it would be necessary to have a test case which was made of a distribution network with the following elements:

- Distribution network with real dimensions

- Information about the tie-lines of the mentioned network 
- The transformers taps

- The steps of the capacitors banks

- Information of the distributed generation.

However, to the best of our knowledge, there are no methods suitable for comparison, since no reconfiguration method has applied to a real-dimension distribution network using jointly all these control devices. Nevertheless, there are many test cases published in the literature, which deal with the reconfiguration problem by tie-switching only, or reconfiguration taking into account the taps of transformers or the reconfiguration using transformers taps and the steps of capacitors banks. We have decided to compare our methodology using two study cases. The first example deals with the DSR problem by switching only and applied to a 32-buses system. The second one is a medium dimension distribution system and the efficiency and strength of the proposed methodology.

\subsection{Example 1}

This test case has been taken from the Reference [1]. It is a hypothetical $12.66 \mathrm{kV}$ system with 2 feeders, one substation, $32 \mathrm{buses,}$ and 5 looping branch (tie-lines). Total substation loads for the base configuration are $5084.26 \mathrm{~kW}$ and $2547 \mathrm{kvar}$. The system is not well-compensated and loss (total loss is about $8 \%$ of the total load). A loss system is selected because loss reduction is expected to be appreciable [7]. The voltage profile of the base system configuration is lower than the usual limit of 0.9 p.u. It shows that the system is not well configured. Also, it is assumed that every branch in the system is available for branch-exchange.

Baran and $\mathrm{Wu}$ [7] have used three different methods (M1, M2, M3) of varying accuracies to estimate the change in loss due to exchange. M1 method consists of the application of Branch Exchanges technique and a simplified power flow used to guide the search process. M2 method is based on the application of the Branch Exchanges technique with the called Backward and Forward Updating of power flow used in Reference [7]. Finally, M3 method applies the Branch Exchanges with an exact power flow. Each switching decision in these methods has been made after checking five switching options corresponding to the five open switches. Total lines loss in the final configuration (M1 method) was found to be of $0.01488578 \mathrm{pu}(148.8578 \mathrm{~kW})$ for a base voltage and power of $12.66 \mathrm{kV}$ and $10 \mathrm{MVA}$, respectively. In the final configuration obtained by Reference [7] open tie-switches are s6, s33, s35, s36, and s37. The voltage profile in the final solution has not been reported in this Reference [7].

Other research works show different results for this same test system. Thus, Reference [35] compares its final losses with those obtained by References [7] and [4]. As starting point of the reconfiguration problem, Reference [36] points out that the loss of the initial configuration is 0.020581 pu. (205.81 kW). In accordance with the Reference [35], the final loss obtained by Reference [7] is $0.01488578 \mathrm{pu}$. (148.8578 kW). On the contrary, in the final configuration obtained by algorithm [4] the lines losses are $0.0141541 \mathrm{pu} .(141.5410 \mathrm{~kW})$ and the open lines are s7, s10, s14, s32, and s37. The solution obtained by Reference [35], gets a loss of 0.01408154 pu. (140.8154 kW) and the open tie-lines are s7, s9, s14, s32, and s37. In this Reference [35] the voltage profile of the initial and final configuration has been reported.

McDermott et al. [37] compare various algorithms, in which the initial and final losses obtained by Reference [7] are 202.68 and $142.60 \mathrm{~kW}$, respectively.

In References [25] and [36] the load conditions of the test case are $5058.25 \mathrm{~kW}$ and $2547.32 \mathrm{kvar}$, which are different from the original values of the Reference [7], from where the test case has been extracted. In this case, the initial losses are 202.68 kW. Gomes et al. [25] have elaborated a methodology and compared their method with References [4,35,36] and [37]. In this Reference [25], the voltage profile has not been reported. The comparison can be observed in Table I, which has been extracted from Reference [25]. To this table has been added the execution time of the methodology proposed in this paper. It is important to notify that the optimum solution has been obtained by force brute algorithm in References $[25,36]$. The same solution has also been obtained by the proposed methodology.

In this paper the problem of DSR integrated with DOPF has been studied for the test case represented in Figure 2, which has been taken from Reference [7] and verified in Reference [35]. The dotted lines in Figure 2 represent the tie-lines. Switches s33, s34, s35, s36, and s37 are open for the initial configuration, and it is assumed that on/off switches exist at every node of the network.

The optimization of the DSR integrated with OPF problem is applied to the network in Figure 3. The power flow is represented in a single direction only if this circulates exclusively from the source toward the load bus. If a doubt about feeding the load nodes from any substation exists, a line with two-way direction is used to represent the power flow, so that each node can be fed from any substation or generator. The switches have been represented by dashed segments. The mathematical model selects the lines in each Benders decomposition iteration.

The data for the network has been extracted from Reference [7] and can be obtained from the authors on request. 
Table I. Comparison with various methodologies from References [25] and [37] and the proposed methodology.

\begin{tabular}{|c|c|c|c|c|}
\hline Method & Final losses $(\mathrm{kW})$ & Saving (\%) & CPU Time (S) & Open switches \\
\hline Optimum & 136.57 & 32.6 & 647.03 & $\mathrm{~s} 7, \mathrm{~s} 9, \mathrm{~s} 14, \mathrm{~s} 32, \mathrm{~s} 37$ \\
\hline Gomes [37] & 136.57 & 32.6 & 1.66 & s7, s9, s14, s32, s37 \\
\hline Goswami [35] & 136.57 & 32.6 & 0.87 & $\mathrm{~s} 7, \mathrm{~s} 9, \mathrm{~s} 14, \mathrm{~s} 32, \mathrm{~s} 37$ \\
\hline McDermott [38] & 136.57 & 32.6 & 1.99 & $\mathrm{~s} 7, \mathrm{~s} 9, \mathrm{~s} 14, \mathrm{~s} 32, \mathrm{~s} 37$ \\
\hline Gomes [25] & 136.66 & 32.5 & 0.96 & $\mathrm{~s} 7, \mathrm{~s} 10, \mathrm{~s} 14, \mathrm{~s} 32, \mathrm{~s} 37$ \\
\hline Shirmohammadi [4] & 136.66 & 32.5 & 0.14 & $\mathrm{~s} 7, \mathrm{~s} 10, \mathrm{~s} 14, \mathrm{~s} 32, \mathrm{~s} 37$ \\
\hline Proposed methodology & 139.55 & 31.1 & 0.11 & s7, s9, s14, s32, s37 \\
\hline
\end{tabular}

The total line losses in the final configuration obtained by the proposed methodology are $0.0139550 \mathrm{pu}$. (139.550 kW) and the open tie-lines are s7, s9, s14, s32, and s37.

After comparing the results obtained by the proposed methodology with others published in the international literature, we could observe that there are incongruities between the results reported by several studies with regard to total active power loss. Nevertheless, with respect to open tie-lines, it can be assured that our results are equal to the results obtained by References [25,35] and [36].

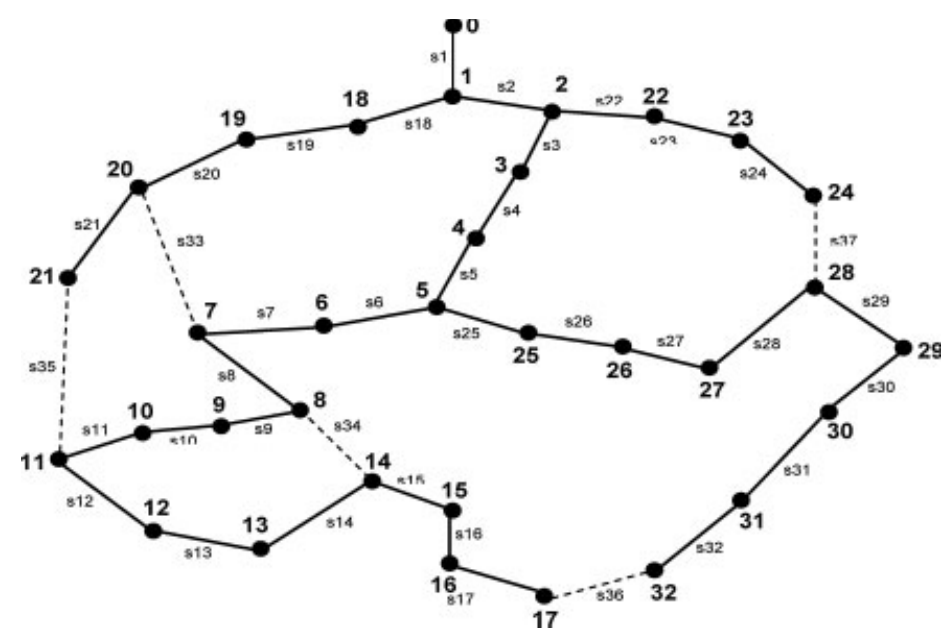

Figure 2. Thirty-three bus test system in References [7] and [35].

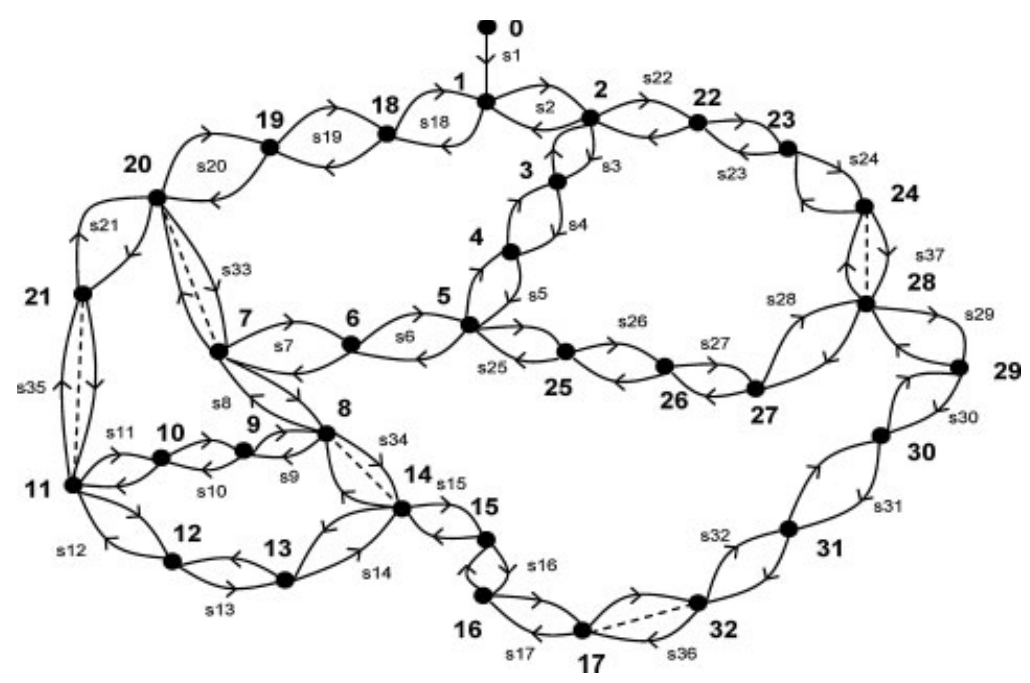

Figure 3. Representation of the distribution network. 
Table II. Comparison with various methodologies applying power flow to all obtained configuration.

\begin{tabular}{lccr}
\hline Method & Final losses $(\mathrm{kW})$ & Saving $(\%)$ & Open switches \\
\hline Optimum [36] & 139.550 & 31.147 & $\mathrm{~s} 7, \mathrm{~s} 9, \mathrm{~s} 14, \mathrm{~s} 32, \mathrm{~s} 37$ \\
Proposed methodology & 139.550 & 31.147 & $\mathrm{~s} 7, \mathrm{~s} 9, \mathrm{~s} 14, \mathrm{~s} 32, \mathrm{~s} 37$ \\
Gomes [37] & 139.550 & 31.147 & $\mathrm{~s} 7, \mathrm{~s} 9, \mathrm{~s} 14, \mathrm{~s} 32, \mathrm{~s} 37$ \\
Goswami [35] & 139.550 & 31.147 & $\mathrm{~s} 7, \mathrm{~s} 9, \mathrm{~s} 14, \mathrm{~s} 32, \mathrm{~s} 37$ \\
McDermott [38] & 139.550 & 31.147 & $\mathrm{~s} 7, \mathrm{~s} 9, \mathrm{~s} 14, \mathrm{~s} 32, \mathrm{~s} 37$ \\
Gomes [25] & 140.278 & 30.787 & $\mathrm{~s} 7, \mathrm{~s} 10, \mathrm{~s} 14, \mathrm{~s} 32, \mathrm{~s} 37$ \\
Shirmohammadi [4] & 140.278 & 30.787 & $\mathrm{~s} 7, \mathrm{~s} 10, \mathrm{~s} 14, \mathrm{~s} 32, \mathrm{~s} 37$ \\
Baran [7] & 146.832 & 27.554 & $\mathrm{~s} 11, \mathrm{~s} 28, \mathrm{~s} 31, \mathrm{~s} 33, \mathrm{~s} 34$ \\
Actual network [25,37] & 202.677 & 00.000 & $\mathrm{~s} 33, \mathrm{~s} 34, \mathrm{~s} 35, \mathrm{~s} 36, \mathrm{~s} 37$ \\
\hline
\end{tabular}

In view of these incongruities, we proceed to investigate the data, which were found exactly coincident with the original data of the above References [7] and [35].

To know what was happening with the resulting incongruities, we took every configuration obtained by those studies and executed a power flow in each and also for the configuration obtained by the proposed methodology. It can be noticed that the results obtained by References [25] and [36] (see Table I) are slightly different from the values presented in Table II, which were obtained in the present study. It is important to emphasize that the values in Table II, have been obtained by using the power flow for every configuration provided by the aforementioned references. Table III shows the feeders current values before and after reconfiguration obtained by References [7,4,25,35-37] and the proposed methodology, respectively.

Moreover, Table IV presents the voltage profile for the initial configuration of Reference [7] and the final configuration of References [4,7,25,35-37] and the proposed methodology. These voltage profiles have been obtained through a power flow for radial distribution systems. All the results are available on request from us.

Finally, it should be noted that the configuration obtained by the proposed algorithm is equal to the results obtained by References [36,37] and seems to represent the optimum by obtaining the minimum power losses and the minimal number of switches opened/closed. Nevertheless, the reconfigured network has a better voltage profile since the minimum voltage is 0.937819 p.u. (node 31), whereas in References [34] and [25] it reaches 0.9232616 p.u. (node 31) and 0.9378185 p.u. (node 31 ), respectively. This case converges on only four iterations and with a computation time of 0.055 seconds, which has been executed in a computer with two Quad-Core Intel ${ }^{\mathbf{1}}$ Xeon $^{\mathbf{1}}$ Processors E5310 Series, $1.6 \mathrm{GHz}$ and 4 GB of RAM with a Windows Server 2003 Operating System.

\subsection{Example 2}

The new system tested is an $11 \mathrm{kV}$ radial distribution system having two substations, 4 feeders, 70 nodes and 78 branches (including tie-lines) as shown in Figure 4. Tie-switches of this system are open in the normal condition. System data are extracted from Appendix A of Reference [1]. They are also available in the online version of this paper as Supporting information.

The formulation of the optimization of reconfiguration problem is based on the network of Figure 4. In this figure, switches have been represented by dashed segments.

In Reference [1] it is mentioned that before distribution network reconfiguration, total real power losses of this system are $337.45 \mathrm{~kW}$ and the minimum voltage is $V_{\min }{ }^{1 / 4} V_{67} 1 / 40.88389$ p.u.

Table III. Comparison of results obtained by the References $[7,4,25,35-38]$ and the proposed methodology.

\begin{tabular}{|c|c|c|c|}
\hline \multicolumn{4}{|c|}{ Feeders currents before and after reconfiguration, values in ampère } \\
\hline $\begin{array}{l}\text { Before } \\
\text { reconfiguration[7] }\end{array}$ & $\begin{array}{c}\text { After reconfiguration } \\
\text { obtained by Reference [7] }\end{array}$ & $\begin{array}{c}\text { After reconfiguration } \\
\text { obtained by References }[4,25]\end{array}$ & $\begin{array}{c}\text { After reconfiguration } \\
\text { obtained by References }[35,36,37,38] \\
\text { and the proposed methodology }\end{array}$ \\
\hline $\operatorname{IF}(1-2) 1 / 4328.77$ & $\mathrm{IF}(1-2) 1 / 4248.63$ & $\mathrm{IF}(1-2) 1 / 4235.51$ & $\operatorname{IF}(1-2) 1 / 4235.51$ \\
\hline $\operatorname{IF}(1-18) 1 / 428.71$ & $\operatorname{IF}(1-18) 1 / 4104.29$ & $\operatorname{IF}(1-18) 1 / 4111.65$ & $\operatorname{IF}(1-18) 1 / 4111.78$ \\
\hline
\end{tabular}


Table IV. Voltage profile obtained by the References [7,4,25,35-38] and the proposed methodology.

\begin{tabular}{|c|c|c|c|c|}
\hline Node & $\begin{array}{l}\text { Initial configuration } \\
\text { of the network [7] }\end{array}$ & $\begin{array}{l}\text { Configuration obtained } \\
\text { by Reference [7] } \\
\text { after reconfiguration }\end{array}$ & $\begin{array}{l}\text { Configuration obtained } \\
\text { by References }[4,25]\end{array}$ & $\begin{array}{c}\text { Configuration obtained } \\
\text { by References [35,36,37,38] } \\
\text { and the proposed methodology }\end{array}$ \\
\hline 0 & 1.0000000 & 1.0000000 & 1.0000000 & 1.0000000 \\
\hline 1 & 0.9970323 & 0.9970708 & 0.9970776 & 0.9970782 \\
\hline 2 & 0.9829380 & 0.9864138 & 0.9869909 & 0.9869915 \\
\hline 3 & 0.9754564 & 0.9835440 & 0.9824720 & 0.9824726 \\
\hline 4 & 0.9680592 & 0.9809444 & 0.9781570 & 0.9781576 \\
\hline 5 & 0.9496582 & 0.9750771 & 0.9673163 & 0.9673168 \\
\hline 6 & 0.9461726 & 0.9733230 & 0.9666750 & 0.9666756 \\
\hline 7 & 0.9413284 & 0.9713967 & 0.9615375 & 0.9626152 \\
\hline 8 & 0.9350594 & 0.9699693 & 0.9576645 & 0.9592472 \\
\hline 9 & 0.9292444 & 0.9690252 & 0.9571597 & 0.9626999 \\
\hline 10 & 0.9283844 & 0.9689557 & 0.9643907 & 0.9627847 \\
\hline 11 & 0.9268848 & 0.9538125 & 0.9645150 & 0.9630796 \\
\hline 12 & 0.9207717 & 0.9440395 & 0.9619468 & 0.9604988 \\
\hline 13 & 0.9185050 & 0.9401454 & 0.9611547 & 0.9597055 \\
\hline 14 & 0.9170927 & 0.9371778 & 0.9515998 & 0.9531927 \\
\hline 15 & 0.9157248 & 0.9339523 & 0.9498405 & 0.9514364 \\
\hline 16 & 0.9136975 & 0.9279670 & 0.9469188 & 0.9485196 \\
\hline 17 & 0.9130905 & 0.9254893 & 0.9458915 & 0.9474941 \\
\hline 18 & 0.9965039 & 0.9952720 & 0.9950750 & 0.9950768 \\
\hline 19 & 0.9929263 & 0.9802671 & 0.9782329 & 0.9782458 \\
\hline 20 & 0.9922218 & 0.9761698 & 0.9735994 & 0.9736156 \\
\hline 21 & 0.9915844 & 0.9693899 & 0.9705426 & 0.9701564 \\
\hline 22 & 0.9793523 & 0.9799069 & 0.9834203 & 0.9834208 \\
\hline 23 & 0.9726811 & 0.9669578 & 0.9767772 & 0.9767778 \\
\hline 24 & 0.9693561 & 0.9574195 & 0.9734662 & 0.9734668 \\
\hline 25 & 0.9477289 & 0.9747968 & 0.9655366 & 0.9655372 \\
\hline 26 & 0.9451652 & 0.9745367 & 0.9631798 & 0.9631804 \\
\hline 27 & 0.9337256 & 0.9740100 & 0.9526576 & 0.9526582 \\
\hline 28 & 0.9255075 & 0.9534489 & 0.9451246 & 0.9451252 \\
\hline 29 & 0.9219501 & 0.9511453 & 0.9419160 & 0.9419166 \\
\hline 30 & 0.9177889 & 0.9497422 & 0.9384931 & 0.9384937 \\
\hline 31 & 0.9168735 & 0.9232616 & 0.9378185 & 0.9378191 \\
\hline 32 & 0.9165898 & 0.9241040 & 0.9455616 & 0.9471647 \\
\hline
\end{tabular}

After application of the method exposed in Reference [1], real power losses are reduced to $302.05 \mathrm{~kW}$ and the minimum voltage rises to $V_{\min } 1 / 4 V_{29} 1 / 40.91214$ p.u.

In this paper, after the application of the proposed methodology, the real power losses of this system are $301.6453 \mathrm{~kW}$ and the minimum voltage is $V_{\min } 1 / 4 V_{29} 1 / 40.915514$ p.u. In order to compare the results of Reference [1] and the proposed methodology, we executed a full power flow in each configuration. Firstly, we took the configuration before and after reconfiguration presented in Reference [1]. The total active power losses in the distribution network before and after reconfiguration are 341.427 and $304.904 \mathrm{~kW}$, respectively. However, the total active power losses obtained by the proposed methodology are $301.645 \mathrm{~kW}$. It can be noticed that the results obtained by Reference [1] and the proposed method are slightly different. This slight difference may be attributed to the use of exact power flow equations in the proposed methodology. Moreover, the feeders currents after the application of this methodology are more balanced than the feeders currents obtained in Reference [1]. The comparison of results between the proposed methodology and the method described in Reference [1] is presented in Table V. Table VI shows the feeders currents values in the both cases.

It should be noted that the algorithm presented in Reference [1] is based on heuristic rules and fuzzy multi-objective approach. The objectives considered attempt to maximize the fuzzy satisfaction of the load balancing among the feeders, minimization of power loss, deviation of the nodes voltage and branch current constraint violation subject to radial network structure.

In our humble opinion, the use of heuristic methods is not always able to obtain an optimal solution. 


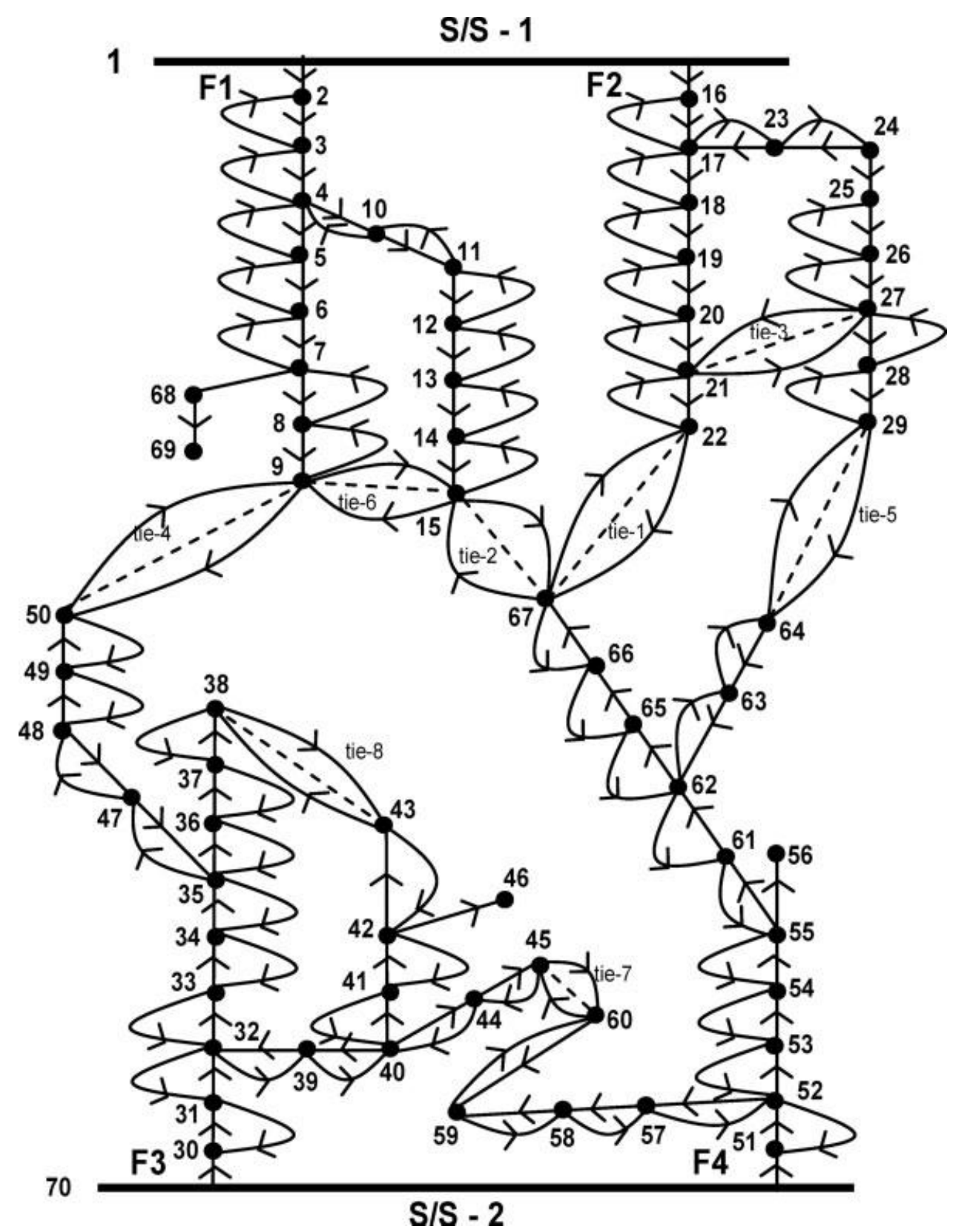

Figure 4. Representation of the distribution network, original extracted from Reference [1].

To prove the efficiency and strength of the proposed methodology, the database extracted from Reference [1] has been modified to comply with the following conditions:

- Distribution network with medium dimensions

- Information about the tie-switches of the network

- The transformers taps

- The steps of the capacitors banks

- The information of the distributed generation 
Table V. Comparison of results Reference [1] and the proposed methodology.

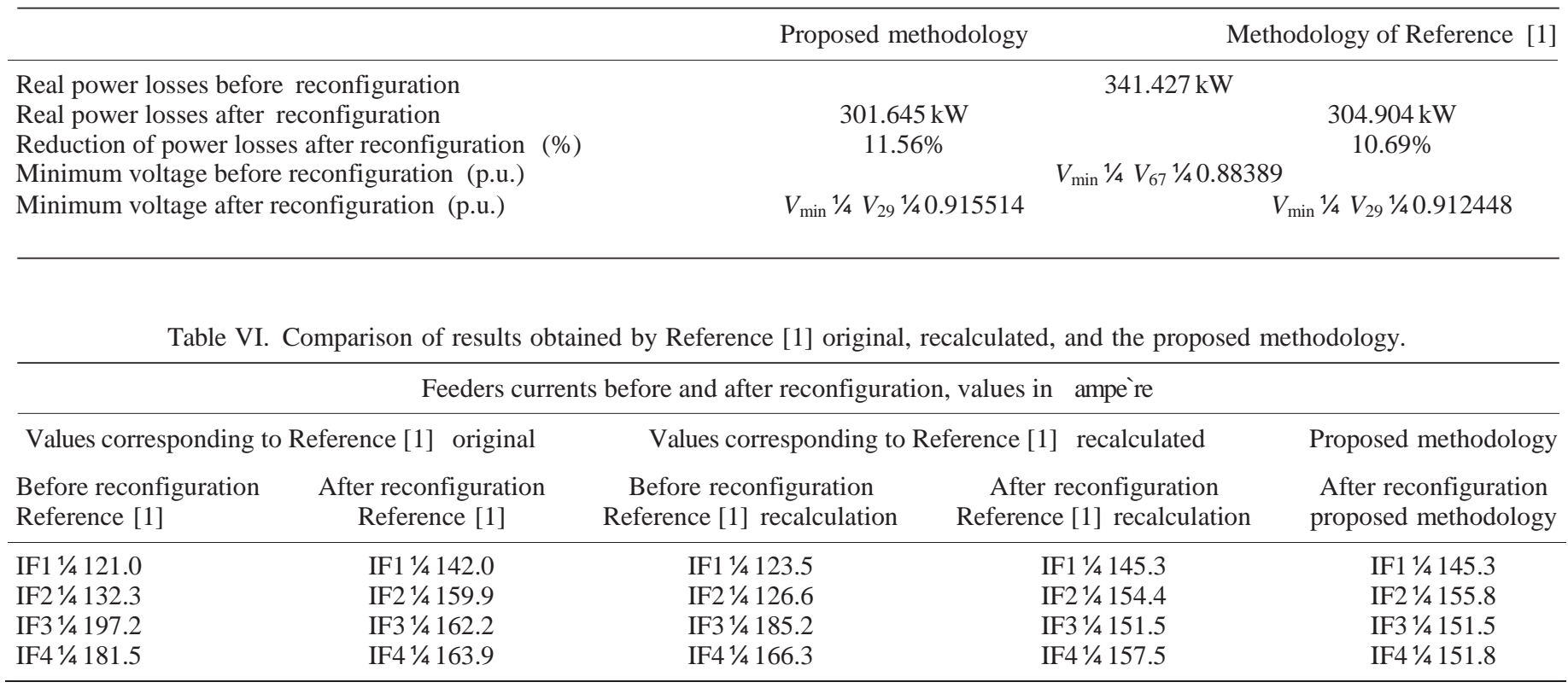

Two capacitors banks have been settled in nodes 49 and 64, each one with three steps: 150, 300, and 600 kvar. The transformers taps have been considered in the two substations, nodes 1 and 70, and bus voltages range from 0.955 to 1.05 p.u. Two generators of 2 MVA each have been installed in nodes 15 and 29. This paper analyzes five cases.

- Case I: reconfiguration considering the tie-switches only.

- Case II: reconfiguration considering transformers taps.

- Case III: reconfiguration considering the capacitor banks steps.

- Case IV: reconfiguration considering taps and capacitor banks.

- Case V: reconfiguration considering taps, capacitors banks and distributed generation.

Table VII presents the detailed calculation of the variables and constraints number of Master and Slave problems for each study case, as well as for the case of Reference [1]. It also shows the computing complexity of the model.

The results of the executed cases can be observed in Tables VII and VIII. It should be noted that the best case (minimum losses) is Case V, that is, the case where the capacitors banks and the distributed generation have been connected into the distribution network and the taps of the distribution substations transformers have been taken into account.

Case I consists of the direct application of the proposed methodology to determine the optimal configuration of the distribution network by tie-switching only. The resulting active power losses are $301.6453 \mathrm{~kW}$. The lower voltage (0.915514 p.u.) is reached at node 29. A single iteration is needed for the convergence and the CPU time is 0.140 seconds.

Table VII. Computation of time execution and number of variables and constraints of Master and Slave problems.

\begin{tabular}{|c|c|c|c|c|c|c|}
\hline \multirow[t]{2}{*}{ Cases } & \multirow[t]{2}{*}{ Number of iterations } & \multirow[t]{2}{*}{ CPU time (seconds) } & \multicolumn{2}{|c|}{ Number of variables } & \multicolumn{2}{|c|}{ Number of constraints } \\
\hline & & & Master & Slave & Master & Slave \\
\hline Case I & 1 & 0.140 & 289 & 642 & 416 & 775 \\
\hline Case II & 1 & 0.142 & 289 & 660 & 373 & 779 \\
\hline Case III & 2 & 0.297 & 297 & 652 & 384 & 785 \\
\hline Case IV & 1 & 0.234 & 297 & 670 & 368 & 789 \\
\hline Case V & 1 & 0.203 & 299 & 662 & 365 & 799 \\
\hline
\end{tabular}


Table VIII. Results of: active power losses, transformers taps position and power generated by capacitors and generators.

\begin{tabular}{|c|c|c|c|c|c|c|c|c|c|}
\hline \multirow[t]{2}{*}{ Cases } & \multirow[t]{2}{*}{ Active power losses $(\mathrm{kW})$} & \multicolumn{4}{|c|}{ Transformer taps (p.u.) } & \multicolumn{2}{|c|}{ Capacitor (kvar) } & \multicolumn{2}{|c|}{ Generator (kVA) } \\
\hline & & $(1-2)$ & $(1-16)$ & $(70-30)$ & $(70-51)$ & Bus 49 & Bus 64 & Bus 15 & Bus 29 \\
\hline Case I & 301.6453 & \multicolumn{4}{|c|}{ Transformers Taps not considered } & \multicolumn{2}{|c|}{ Not considered } & \multicolumn{2}{|c|}{ Not considered } \\
\hline Case II & 301.6453 & 1.003 & 1.002 & 1.006 & 1.006 & \multicolumn{2}{|c|}{ Not considered } & \multirow{3}{*}{\multicolumn{2}{|c|}{$\begin{array}{l}\text { Not considered } \\
\text { Not considered } \\
\text { Not considered }\end{array}$}} \\
\hline Case III & 287.142 & & Not & isidered & & 600 & 150 & & \\
\hline Case IV & 287.142 & 0.999 & 1.029 & 1.001 & 1.001 & 600 & 150 & & \\
\hline Case V & 196.810 & 1.007 & 1.016 & 1.003 & 1.004 & 0 & 300 & 372 & 561 \\
\hline
\end{tabular}

Table IX. Comparison of opened branches in each study case.

\begin{tabular}{|c|c|c|c|c|c|}
\hline \multirow[t]{2}{*}{ Results of Reference [1] } & \multicolumn{5}{|c|}{ Cases studied by the proposed methodology } \\
\hline & Case I & Case II & Case III & Case IV & Case V \\
\hline $9-15$ & $9-15$ & $9-15$ & $6-7$ & $6-7$ & $7-8$ \\
\hline $15-67$ & $15-67$ & $15-67$ & $9-15$ & $9-15$ & $14-15$ \\
\hline $26-27$ & $21-27$ & $21-27$ & $15-67$ & $15-67$ & $15-67$ \\
\hline $29-64$ & $28-29$ & $28-29$ & $21-27$ & $21-27$ & $21-27$ \\
\hline $38-43$ & $37-38$ & $37-38$ & $28-29$ & $28-29$ & $27-28$ \\
\hline $44-45$ & $40-44$ & $40-44$ & $37-38$ & $37-38$ & $37-38$ \\
\hline $49-50$ & $49-50$ & $49-50$ & $40-44$ & $40-44$ & $40-44$ \\
\hline $65-66$ & $62-65$ & $62-65$ & $62-65$ & $62-65$ & $48-49$ \\
\hline- & - & - & - & - & $55-61$ \\
\hline - & - & - & - & - & $62-65$ \\
\hline
\end{tabular}

Case II presents the application of the proposed methodology taking into account the taps of the distribution substations transformers, which are in nodes 1 and 70. The number of variables and equations involved can be seen in Table VII. Real power losses are $301.6453 \mathrm{~kW}$. This case converges on a single iteration and with a CPU time of 0.142 seconds. The worst node is 29 , with a voltage of 0.92655 p.u. Note that in cases I and II, we obtained the same configuration of the distribution network, but with the consideration of the transformers taps, the worst node voltage is higher.

Case III presents the application of the proposed methodology taking into account three steps for each capacitors bank. These capacitors are installed in nodes 49 and 64 and each contains three steps of 150, 300, and 600 kvar. The active power losses rise to $287.142 \mathrm{~kW}$. A single iteration is needed for the convergence and the time computation is 0.297 seconds. The worst node is the number 69 with a voltage of 0.9113023 p.u. The selected capacitors are: 600 kvar connected in node 49 and 150 kvar connected in node 64.

Case IV applies the proposed methodology considering the taps of transformers and three steps of every capacitors bank. In this study case, the active power losses are $287.142 \mathrm{~kW}$. It converges on a single iteration and with a time of 0.234 seconds. The worst node is number 47 with a voltage magnitude of 0.923564 p.u. Note that in cases III and IV, we obtained the same configuration of the distribution network, but using both resources (capacitors and taps), the worst node voltage is higher.

Finally, Case V studies the optimal configuration of the distribution network taking into account transformers taps, capacitors banks and distributed generation located at buses 15 and 29. The active power losses are $196.810 \mathrm{~kW}$. This case needs a single iteration for the convergence $(0.203$ seconds). The worst node voltage is 65 with a 0.9227526 p.u. The selected capacitor is a 300 kvar connected in node 64.

Finally, Table IX shows the final radial configuration of the five distribution networks obtained for each studied case.

\section{CONCLUSION}

In this paper, we have presented a new and efficient methodology for optimal reconfiguration of distribution networks integrated with an optimal power flow and solved by Benders decomposition optimization technique. The objective minimizes the active and 
reactive power loss modules, balancing load among the feeders and subject to constraints as: the capacity limit of the branches, minimum and maximum limits of the substations or generators, minimum nodes voltages and radial operation of the network.

A novel variant of the generalized Benders decomposition algorithm was applied for solving the problem. The formulation has been embedded under two stages. The first one is the Master problem and is formulated as mixed integer quadratic programming. This stage determines the radial topology of the distribution network. The second stage is the Slave problem and is formulated as non-linear programming. This stage is used to determine the feasibility of the Master problem solution by means of an OPF and provides information for formulating the linear Benders cuts, which couple both stages.

The application of the methodology to two cases extracted from specialized literature demonstrates the effectiveness and robustness of the proposal. It is also verified that the model needs very low execution time for solving the whole problem, a mixedinteger quadratic programming (Master problem) and also a non-linear programming problem (Slave problem). It also seems to be suitable for an application in real time.

\section{LIST OF SYMBOLS AND ABBREVIATIONS}

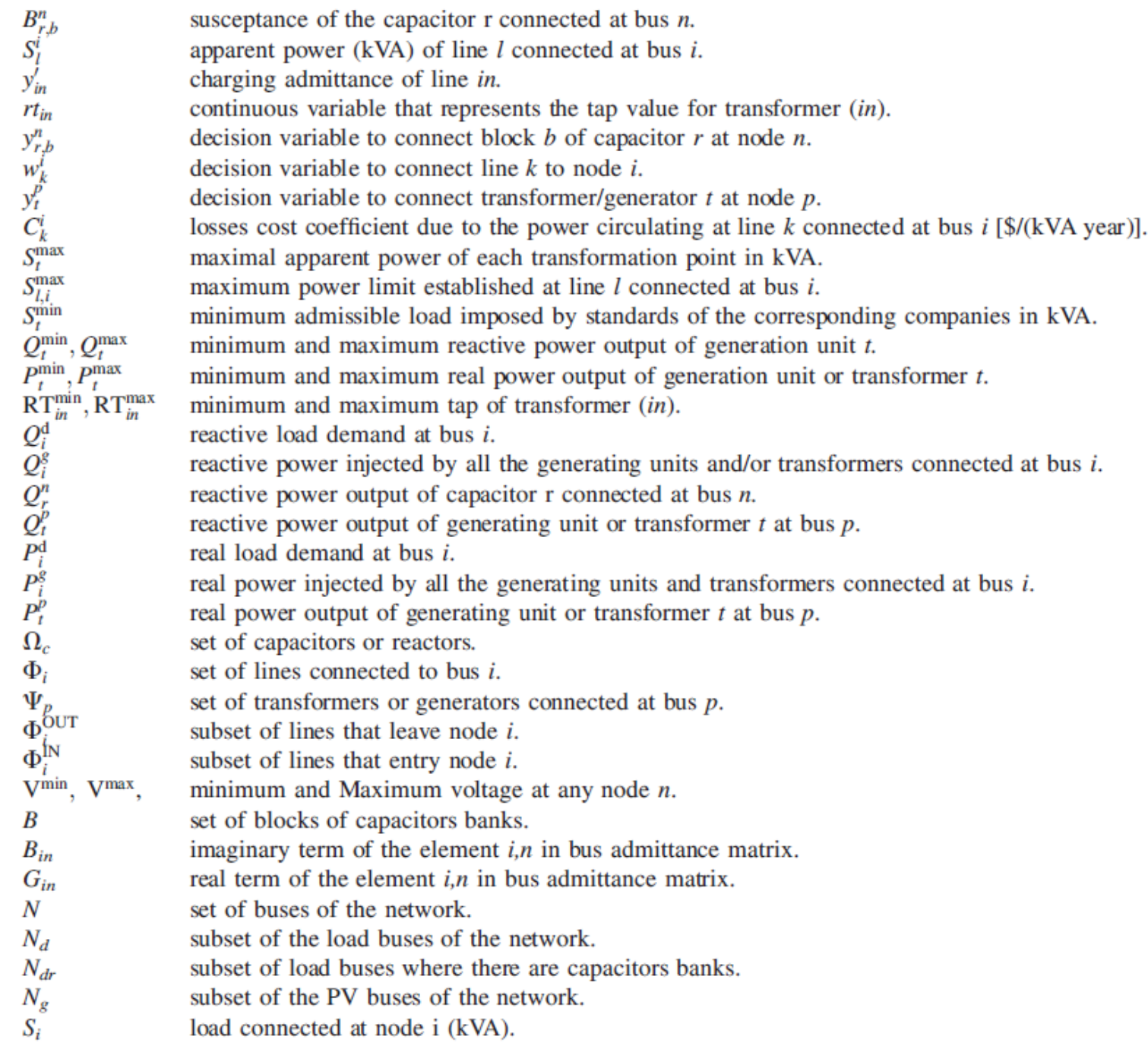




$\begin{array}{ll}V_{n} & \text { bus voltage at bus } n . \\ V_{n} & \text { bus voltage magnitude at bus } n . \\ y_{i n} & \text { series admittance of line in. } \\ \delta_{n} & \text { phase angle at bus } n .\end{array}$

\section{ACKNOWLEDGEMENTS}

H.M. Khodr, Z. A. Vale, and C. Ramos would like to acknowledge FCT, FEDER, POCTI, POSI, POCI, POSC, and PTDC for their support to R\&D Projects and GECAD unit.

\section{REFERENCES}

1. Das D. Reconfiguration of distribution system using fuzzy multi-objective approach. International Journal of Electrical Power \& Energy Systems 2006; 28(5):331-338.

2. Sarfi RJ, Salama MMA, Chikhani AY. A survey of the state of the art in distribution system reconfiguration for system loss reduction. Electric Power Systems Research 1994; 31(1):61-70.

3. Merlin A, Back H. Search for a minimal-loss operating spanning tree configuration in an urban power distribution system. Proceedings of the 5Th Power System Computation Conference, Cambridge, UK, 1975; 1-18.

4. Shirmohammadi D, Hong HW. Reconfiguration for electric distribution networks for resistive line loss reduction. IEEE Transactions on Power Delivery 1989; 4:1492-1498.

5. Borozan V, Rajicic D, Ackovski R. Improved method for loss minimization in distribution networks. IEEE Transactions on Power Systems 1995; 10:1420-1425.

6. Civanlar S, Grainger JJ, Yin H, Lee SSH. Distribution feeder reconfiguration for loss reduction. IEEE Transactions on Power Delivery 1988; 3:1217-1223.

7. Baran ME, Wu FF. Network reconfiguration in distribution systems for loss reduction and load balancing. IEEE Transactions on Power Delivery 1989; 4:14011407.

8. Kashem MA, Ganapathy V, Jasmon GB. Network reconfiguration for enhancement of voltage stability in distribution networks. IEE Proceedings Generation, Transmission \& Distribution 2000; 147(3):171-175.

9. Chen CS, Cho MY. Energy loss reduction by critical switches. 1993; IEEE Transactions on Power Delivery 8:1246-1253.

10. Nara K, Satoh T, Kitagawa M. Distribution system loss minimum reconfiguration by genetic algorithm. Proceedings of the 3rd Symposium on Expert Systems Applications to Power Systems (ESAPS), Tokyo and Kobe, Japan, 1991; 724-730.

11. Lin WM, Cheng FS, Tsay MT. Distribution feeder reconfiguration with refined genetic algorithm. IEE Proceedings: Generation, Transmission \& Distribution $2000 ; 147(6): 349-354$

12. Huang YC. Enhanced genetic algorithm-based fuzzy multi-objective approach to distribution network reconfiguration. IEE Proceedings: Generation, Transmission \& Distribution 2002; 149(5):615-620.

13. Lin WM, Chin HC. A new approach for distribution feeder reconfiguration for loss reduction and service restoration. IEEE Transactions on Power Delivery 1998; 13:870-875

14. Lin WM, Chin HC, Yu GJ. An effective algorithm for distribution feeder loss reduction. Proceedings of the IEEE International Conference on Power System Technology (POWERCON'98), Beijing, China, 1998; Vol. 1, 228-232.

15. Huang KY, Chin HC. Distribution feeder energy conservation by using heuristics fuzzy approach. International Journal of Electrical Power \& Energy Systems $2002 ; 24(6): 439-445$.

16. Liu CC, Lee SJ, Venkata SS. An expert system operational aid for restoration and loss reduction of distribution systems. IEEE Transactions on Power Systems 1988; 3:619-626.

17. Jung KH, Kim H, Ko Y.Network reconfiguration algorithm for automated distribution system based on artificial intelligence approach. IEEE Transactions on Power Delivery 1941; 8:1933-1993.

18. Auguliaro A, Dusonchet L, Ippolito MG, Saneverino ER. Minimum losses reconfiguration of MV distribution networks through local control of tie-switches, IEEE Trans. Power Delivery 2003; 18:762-771.

19. Hsiao YT. Multiobjective evolution programming method for feeder reconfiguration. IEEE Transactions on Power Systems 2004; 19:594-599.

20. Matos MA, Melo P. Multiobjective reconfiguration for loss reduction and service restoration using simulated annealing. Proceedings of the Power Tech'99, Budapest, Hungary, August 1999; 213.

21. Matos MA, Melo P. Loss minimization in distribution networks with multiple load scenarios. Proceedings of the IEEE Power Tech'2001, Porto, Portugal, 2001; Vol. 3.

22. Matos MA, Leao MT, Saraiva JT, et al. Meta-heuristics applied to power systems. In Meta-heuristics: Computer Decision-Making. Kluwer Academic: Norwell, MA, USA, 2003; 449-464.

23. Pereira J, Saraiva JT, Ponce de Leao MT. Identification of operation strategies of distribution networks using a simulated annealing approach. Proceedings of the IEEE Power Tech'99, Budapest, Hungary, August 1999.

24. Ghosh S, Das D. Method for load-flow solution of radial distribution networks. IEE Proceedings: Generation, Transmission and Distribution 1999; $146: 641-648$.

25. Gomes FV, Carneiro S, Pereira JLR, Vinagre MP, Garcia PAN, Araujo LR. A new distribution system reconfiguration approach using optimum power flow and sensitivity analysis for loss reduction. IEEE Transactions on Power Systems 2006; 21(4):1616-1623.

26. Benders JF. Partitioning procedures for solving mixed-variables programming problems. Numerische Mathematik 1962; 4:238-252.

27. Geoffrion AM. Generalized Benders decomposition. Journal of Optimization Theory and Applications 1972; 10(4):237-260.

28. Kagan N, Adams RN. A Benders' decomposition to multi-objective distribution planning problem. International Journal of Electrical Power \& Energy Systems $1993 ; 15(5): 259-271$

29. Alguacil N, Conejo AJ. Multiperiod optimal power flow using Benders decomposition. IEEE Transactions on Power Systems 2000; 15:196-201.

30. Yamin HY, Al-Tallaq K, Shahidehpour SM. New approach for dynamic optimal power flow using Benders decomposition in a deregulated power market. Electric Power Systems Research 2003; 65(2):101-107. 
31. Mart' inez-Crespo J, Usaola J, Fernández JL. Security-constrained optimal generation scheduling in large-scale power systems. IEEE Transactions on Power Systems 2006; 21:321-332.

32. Brooke A, Kendrick D, Meeraus A, Raman R. Release 2.50 GAMS A User Guide. GAMS Development Corp: Washington, DC, 1998.

33. CONOPT and CONOPT2, Bagsvaerd, Denmark: ARKI Consulting and Development, 2001

34. GAMS/CONOPT, Bagsvaerd, Denmark: ARKI Consulting and Development A/S, 2001.

35. Goswami SK, Basu SK. A new algorithm for the reconfiguration of distribution feeders for loss minimization. IEEE Transactions on Power Delivery 1992; 7:1484-1491.

36. Morton AB, Mareels IMY. An efficient brute-force solution to the network reconfiguration problem. IEEE Transactions on Power Delivery 2000; 15(3):9961000 .

37. Gomes FV, Carneiro S, Pereira JLR, Vinagre MP, Garcia PAN, Araujo LR. A new heuristic reconfiguration algorithm for large distribution systems. IEEE Transactions on Power Systems 2006; 20(3):1373-1378.

38. McDermott TE, Drezga I, Broadwater RP. A heuristic nonlinear constructive method for distribution system reconfiguration. IEEE Transactions on Power Systems 1999; 14(2):478-483. 